Communication

\title{
Fabrication of Electrochromic Devices Using Double Layer Conducting Polymers for Infrared Transmittance Control
}

\author{
Jin Kyu Kim, Jong Kwan Koh, Bumsoo Kim², Seokwoo Jeon², Joonmo Ahn , Jong Hak Kim,* \\ ${ }^{1}$ Department of Chemical and Biomolecular Engineering, Yonsei University, 262 Seongsanno, Seodaemum-gu, Seoul 120-746, \\ Republic of Korea, \\ ${ }^{2}$ Department of Materials Science and Engineering, Korea Advanced Institute of Science and Technology (KAIST), 335 \\ Gwahangno, Yuseong-gu, Daejeon 305-701, Republic of Korea \\ ${ }^{3}$ Agency for Defense Development (ADD), Yuseong-gu, Daejeon, 305-152, Republic of Korea
}

\begin{abstract}
We report the performance improvement of electrochromic devices for modulating the transmittance contrast of long wavelength infrared light between 1.5 and $5.0 \mu \mathrm{m}$ based on a double layer of conducting polymers. The device, fabricated with poly(3-hexylthiophene) (P3HT) and poly(3,4-ethylenedioxythiophene) (PEDOT) as the first and second layers, respectively, showed an transmittance contrast of $60 \%$ with a response rate under $5 \mathrm{~s}$, which is greater than the transmittance contrast of cells based on only P3HT or PEDOT (approximately 40\%).
\end{abstract}

Electrochromism is defined as a reversible and visible change in the transmittance of a material by applying different voltages or electric currents, resulting in electrochemical oxidation or reduction [1]. Over the last several decades, a number of electrochromic devices based on various electrochromic materials such as tungsten oxide or nickel oxide have been discovered [2-6]. Electrochromic materials with the ability to modulate radiation in the visible and near infrared wavelengths have been used for optical displays, smart windows, and rear-view mirrors $[7,8]$. However, there have been few studies on infrared (IR) electrochromism, probably due to the relative difficulty in preparing IR transparent electrodes and the lack of interest in IR electrochromic devices [9].

Conducting polymer, known as $\pi$-conjugated polymers, have been extensively investigated due to their potential for application in various areas such as actuators [11], sensors [12], and light-emitting diodes [13]. In particular, conducting polymers have recently gained much attention for use in electrochromic devices because of their excellent coloration efficiency, rapid color-switching ability, and broad color availability $[14,15]$. A range of colors can be controlled and depend on the oxidized (doped) and reduced (undoped) states of the conducting polymers; thus, electroactive conducting polymers have the potential to be electrochromic materials.

Here, we fabricated electrochromic devices using two kinds of conducting polymers, i.e., poly $(3,4-$ ethylenedioxythiophene) (PEDOT) and poly(3hexylthiophene) (P3HT). These materials were made into thin films to modulate the transmittance of IR light between 1.5 and $5.0 \mu \mathrm{m}$. It has been known that PEDOT changes in

*To whom correspondence should be addressed. E-mail: jonghak@yonsei.ac.kr color from faint blue (in its oxidized state) to deep blue (in the reduced state), while P3HT changes from transparent (in its oxidized state) to red-pink (in the neutral state) in the visible range. However, IR electrochromism of these materials has been poorly reported. In this work, double layers of conducting polymers were applied to the device and compared with single layer device performance. The transmittance contrast of electrochromic devices was measured under alternating potential steps $( \pm 2.7 \mathrm{~V})$ in the infrared region between 1.5 and $5.0 \mu \mathrm{m}$.

The electrochromic device structure is made up of a double polished $\mathrm{Si}$ wafer/patterned $\mathrm{Au}$ grid/conducting polymer/liquid electrolyte with spacer/patterned $\mathrm{Au}$ grid/double polished Si wafer, as shown in Fig. 1a. The use of a double polished $\mathrm{Si}$ wafer as a substrate is essential due to its transparency to IR light. In order for electrons to travel in the device, an electrically conducting material should be coated on the wafer substrate. However, most electrically conducting materials, such as metal, carbon, indium tin oxide (ITO), and F-doped tin oxide (FTO), are not transparent to the IR spectral region. Thus, the metal patterned grid was fabricated using $\mathrm{Au}$ via a photolithographic method to minimize the IR light absorption of the substrate (Fig. 1b). The Au patterned Si wafer, with a line width of $20 \mu \mathrm{m}$ and a line repeat period of $500 \mu \mathrm{m}$, showed an IR transmittance of $90 \%$.

Conducting polymer thin films (Fig. 1c) on the Au grid/Si wafer were prepared as an electrochromic material via solution polymerization. For example, PEDOT thin films were formed by spin coating the solution, which consisted of 3,4-ethylenedioxythiophene monomer, pyridine retardant, $\mathrm{Fe}(\mathrm{III})$-tosylate oxidant, and 1-butanol as the solvent. After spin coating, polymerization was carried out at $80^{\circ} \mathrm{C}$ for 5 min. The electrolyte consisted of $1.0 \mathrm{M} \mathrm{LiClO}_{4}$ in propylene carbonate, with a spacer between the $\mathrm{Au}$ grid/Si wafer. The thickness of the electrolyte was controlled by the thickness of the spacer.

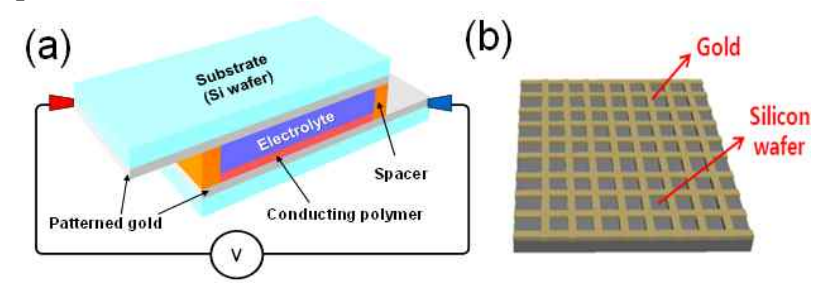


(c)

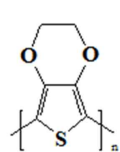

PEDOT

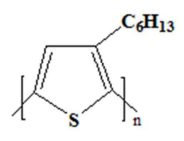

P3HT (d)
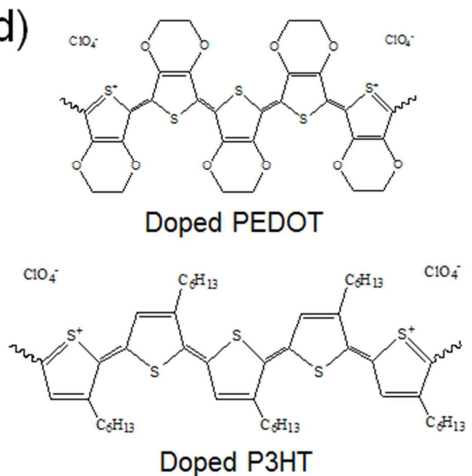

Doped P3HT
Figure 1. (a) Schematic representation of the transmissive infrared electrochromic device, (b) gold patterned silicon wafer, and chemical structures of (c) PEDOT and P3HT, (d) doped PEDOT and doped P3HT.

The IR transmittance spectra of electrochromic devices fabricated with a single layer of conducting polymer are shown in Fig. 2a,b depending on the voltage applied: the neutral state $(-2.7 \mathrm{~V})$ and the doped state $(2.7 \mathrm{~V})$ for PEDOT and P3HT. For both conducting polymer systems, the IR transmittance was reduced in the doped state, compared to the neutral state. This difference is believed to result from the decreased number of carbon double bonds due to the loss of electrons. The transmittance difference between the neutral and the doped states originated from the formation of free charge carriers caused by electrochemical doping and the resultant increase in conduction. The transmittance contrast is plotted based on the transmittance difference between the neutral and doped states between the wavelengths of 1.5 and $5.0 \mu \mathrm{m}$, as shown in Fig. 2c, d. The transmittance contrast of the PEDOT-based device was relatively stable (approximately $40 \%$ over the entire wavelength), but the transmittance contrast of the $\mathrm{P} 3 \mathrm{HT}$ device was strongly dependent on the wavelength. The overall transmittance contrast of the PEDOT-based device was higher than that of P3HT device, probably due to the higher conductivity of the former $(44.4 \mathrm{~S} / \mathrm{cm})$ compared to the latter $(0.1 \mathrm{~S} / \mathrm{cm})$, as measured by an impedance analyzer.

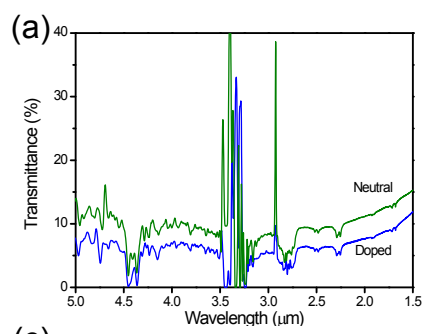

(c)

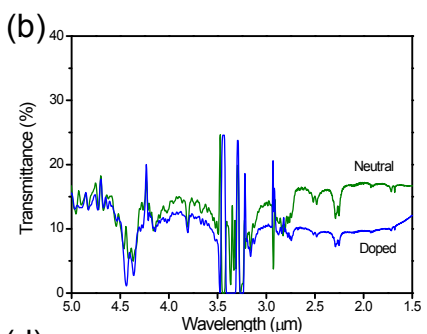

(d)
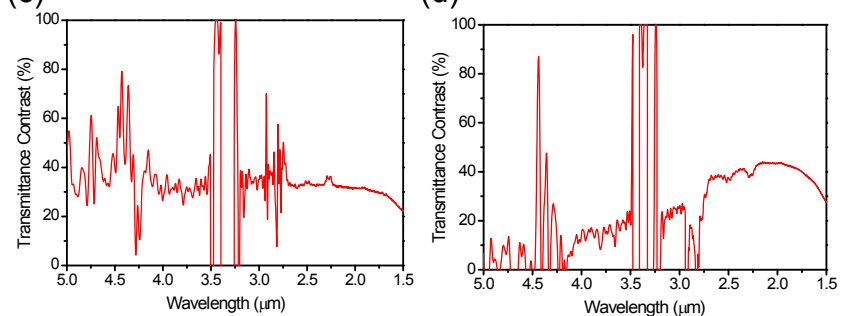

Figure 2. Transmittance spectra of electrochromic devices fabricated with a single layer of conducting polymer: (a) PEDOT and (b) P3HT. The transmittance contrast of (c) PEDOT and (d) P3HT.

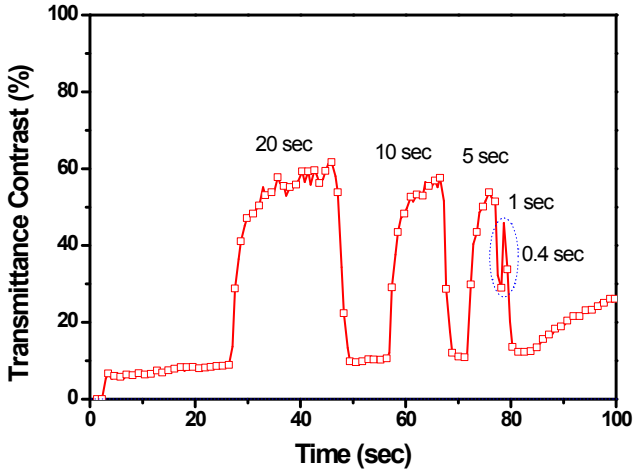

Figure 3. Transmittance contrast of electrochromic device fabricated with double layer PEDOT/P3HT on Au-patterned silicon wafer under alternating potential steps $( \pm 2.7 \mathrm{~V})$.

The electrochromic devices were also fabricated with double layer thin films using P3HT and PEDOT as the first and second layers, respectively. In this scenario, the device configuration was $\mathrm{Si}$ wafer/Au grid/P3HT/PEDOT/electrolyte/Au grid/Si wafer. As shown in Fig. 3, the average transmittance contrast of the double layer cell reached $60 \%$ in the infrared region between 1.5 and 5.0 $\mu \mathrm{m}$, which is much higher compared to the single layer cells. The kinetics of the electrochromic device were also measured using real-time FT-IR (while a square wave voltage between $-2.7 \mathrm{~V}$ and $2.7 \mathrm{~V}$ was applied to the device). The switching time of the undoping reaction was slower than that of the doping reaction, which is consistent with previous reports $[16,17]$. When the half periods of the devices were reduced, the transmittance contrast was slightly reduced, indicating the response time of the cell.

In conclusion, we have developed an IR transmissive electrochromic device using double layer conducting polymer thin films. The device configuration made up of a double polished $\mathrm{Si}$ wafer/patterned $\mathrm{Au}$ grid/P3HT/PEDOT/electrolyte/patterned $\mathrm{Au}$ grid/ double polished Si wafer was suggested. The transmittance contrast of the double layer cell reached $60 \%$ in the infrared region between 1.5 and $5.0 \mu \mathrm{m}$, which is much higher compared to single layer cells. These IR transmissive electrochromic devices have potential for use in military camouflage.

KEYWORDS: electrochromic; conducting polymer; transmittance; double layer; infrared.

Received June 6, 2014; Accepted June 19, 2014

\section{ACKNOWLEDGEMENT}

This work has been supported by the Low Observable Technology Research Center program of the Defense Acquisition Program Administration and the Agency for Defense Development.

\section{REFERENCES AND NOTES}

1. T. Niwa, O. Takai, Thin Solid Films, 2010, 518, 5340.

2. C.J. Brabec, N.S. Sariciftci, J.C. Hummelen, Adv. Funct. Mater. 2001, 11, 15.

3. D. Li, J.X. Huang, R.B. Kaner, Acc. Chem. Res. 2009, 42, 135. 
4. G. Kimura, K. Yamada, Synth. Metals, 2009, 159, 914.

5. K. Yamada, K. Seya, G. Kimura, Synth. Metals, 2009, 159, 188.

6. S. Wu, C. Jia, X. Fu, X. Weng, J. Zhang, L. Deng, Electrochim. Acta, 2013, 88, 322.

7. R.D. Rauh, Electrochim. Acta 1999, 44, 3165.

8. P. Chandrasekhar, B.J. Zay, T. McQueeney, G.C. Birur, V. Sitaram, R. Menon, M. Coviello, R.L. Elsenbaumer, Synth. Metals, 2005, 155, 623.

9. E.B. Franke, C.L. Trimble, J.S. Hale, M. Schubert, J.A. Woollam, J. Appl. Phys. 2000, 88, 5777.

10. B. Kim, J.K. Koh, J.H. Kim, J. Ahn, S. Jeon, Electrochim. Acta. submitted.
11. T.F. Otero, I. Boyano, M.T. Cortes, G. Vazquez, Nucleation, Electrochim. Acta, 2004, 49, 3719.

12. U. Lange, N.V. Roznyatovskaya, V.M. Mirsky, Analy.Chim. Acta, 2008, 614, 1.

13. T.Y. Wu, Y. Chen, J. Polym. Sci. A: Polym. Chem. 2003, 41, 1444.

14. K.-H. Chang, H. P. Wang, T.-Y. Wu, I.-W. Sun, Electrochim. Acta, 2014, 119, 225.

15. P. Camurlu, C. Gultekin, Z. Bicil, Electrochim. Acta, 2012, 61, 50 .

16. V. Jain, H. M. Yochum, R. Montazami, J. R. Heflin, Appl. Phys. Lett. 2008, 92, 033304.

17. M. Kateb, V. Ahmadi, M. Mohseni, Sol. Energy Mater. Sol. Cel. 2013, 112, 57. 